\title{
Does Education Affect HIV Status? Evidence from five African Countries
}

\begin{abstract}
Damien de Walque
Data from the first five Demographic and Health Surveys to include HIV testing for a representative sample of the adult population are used to analyze the socioeconomic correlates of HIV infection and associated sexual behavior. Emerging from a wealth of country relevant results, some important findings can be generalized. First, successive marriages are a significant risk factor. Second, contrary to prima facie evidence, education is not positively associated with HIV status. However, schooling is one of the most consistent predictors of behavior and knowledge: education level predicts protective behaviors such as condom use, use of counseling and testing, discussion of AIDS between spouses, and knowledge about HIV/AIDS, but it also predicts a higher level of infidelity and a lower level of abstinence. JEL codes: I12, O12, O15
\end{abstract}

The HIV/AIDS epidemic is one the greatest challenges facing Africa. According to UNAIDS (2007), in 200720.9 million to 24.3 million people were infected with HIV/AIDS in Sub-Saharan Africa (about 67 percent of the world total), 1.5 million to 2.0 million died from the virus, and 1.4 million to 2.4 million became newly infected.

The socioeconomic profile of the HIV/AIDS epidemic has been analyzed in the epidemiologic and the economics literature. Few of these studies used nationally representative samples. Data sets that include the results of individual HIV tests are generally drawn from cohort studies limited to a specific area (Nunn and others 1994; de Walque 2003, 2007a; de Walque and others 2005), from surveillance data collected from pregnant women attending antenatal care clinics (Fylkesnes and others 1997; Kilian and others 1999), or from high-risk groups (Nagot and others 2002). Some of these data sets have only a limited number of sociodemographic variables, and most cannot claim to be representative.

There are, however, a few studies that use more representative data sets. Fylkesnes and others (2001) compare results from surveillance data among pregnant women and from population-based surveys in Zambia and,

Damien de Walque (corresponding author) is an economist in the Development Research Group at the World Bank; his email address is ddewalque@worldbank.org.

THE WORLD BANK ECONOMIC REVIEW, VOL. 23, NO. 2, pp. 209-233

doi:10.1093/wber/lhp005

Advance Access Publication June 16, 2009

(C) The Author 2009. Published by Oxford University Press on behalf of the International Bank for Reconstruction and Development / THE WORLD BANK. All rights reserved. For permissions, please e-mail: journals.permissions@oxfordjournals.org 
in both data sources, observe trends showing large declines in HIV prevalence associated with higher educational background and stable or rising prevalence associated with low education.

This study goes further by using data from the first five Demographic and Health Surveys to include data on HIV testing for a nationally representative sample of the adult population. The data sets are from Burkina Faso (2003), Cameroon (2004), Ghana (2003), Kenya (2003), and Tanzania (2003-04), five African countries with HIV/AIDS epidemics of different proportions. HIV prevalence is substantially higher in Cameroon, Kenya, and Tanzania than in Burkina Faso and Ghana. The five data sets have very similar variables, allowing easy comparisons across countries. ${ }^{1}$ They also include a large set of sociodemographic variables and numerous questions about sexual behavior and other practices and attitudes related to the AIDS epidemic. ${ }^{2}$

These five data sets are used to analyze the socioeconomic determinants of HIV infection in the general population, looking at the association between HIV status and urban status, marital status, education, wealth, and religion. Also analyzed are the associations between these factors and a large range of sexual behaviors and other practices and attitudes related to the HIV/AIDS epidemic, allowing for a better understanding of the channels through which socioeconomic variables can affect HIV infection. The analysis also focuses specifically on the relationship between education and sexual behaviors and attitudes associated with HIV/AIDS.

The detailed description of the results provides substantial information relevant at the country level and gives a comparative view of the HIV/AIDS epidemic. While some differences across countries are to be expected, the results across countries are surprisingly consistent. Having similar information about five African countries for the same period permits using both pooled and country-level regressions to assess which of the results can be generalized and are broadly relevant for policymakers engaged in the fight against the epidemic.

Successive marriages is found to be a significant risk factor, as evidenced by the association with HIV prevalence and sexual behavior, suggesting that specific prevention efforts should be targeted to this group.

Further, and contrary to prima facie evidence, education is not positively associated with HIV status. But schooling is one of the most consistent predictors of behavior and knowledge. Education predicts protective behaviors such as condom use, use of counseling and testing, discussion between spouses, and knowledge about HIV/AIDS, but it also predicts a higher level of infidelity and a lower level of abstinence. It is possible that these associations, moving in

1. Buvé, Caraël, and Hayes (2001) describe an interesting multicenter study of risk factors for HIV infection in four cities in different African countries.

2. Gersovitz (2005) provides a useful discussion of the variables describing sexual behavior in Demographic and Health Surveys. 
opposite directions, cancel each other out and that, as a consequence, education is not significantly associated with HIV status. However, while it is difficult to isolate a significant relationship between education and HIV in the overall population, a negative association can be found in urban settings, at least in regressions that pool the data from the five countries.

Section I describes the data sets and the methodology. Section II covers the analysis of HIV status. Section III focuses on the association between education and a large range of sexual behaviors and other attitudes related to the epidemic. Section IV presents implications for further research and for policymakers.

\section{Data Description and Methodology}

The five data sets used are very similar: four of them (Burkina Faso, 2003; Cameroon, 2004; Ghana, 2003; and Kenya, 2003) are standard Demographic and Health Surveys that also include data on HIV testing for a subsample of the population (Burkina Faso and ORC Macro 2004; Cameroon and ORC Macro 2004; Ghana and ORC Macro 2004; and Kenya and ORC Macro 2004). The 2003-04 HIV/AIDS Indicator Survey for Tanzania is a lighter survey that focuses on HIV/AIDS, but for the purposes of this study, the relevant variables are very similar (Tanzania and ORC Macro 2005).

The independent variables used in the regressions are almost always the same: urban location, marital status, including polygamy and successive marriages, education, proxies for wealth and poverty, and religion. ${ }^{3}$ Summary statistics are in table 1 . Not shown in the tables but included in the regression are dummy variables for age, region, and ethnicity (except for Tanzania, for which the ethnicity variable is not available).

The share of the urban population is much higher in Cameroon and Ghana than in the other three countries (table 1). Educational achievement, measured by the highest grade achieved, is generally higher for men than for women and is much lower in Burkina Faso than in the other countries. Several variables describe marital status. The omitted category comprises individuals who have never been married. Marriage is defined as being legally married or living with a partner with the intention of staying together and therefore covers both formal and informal marriage. The formerly married category includes widowed, divorced, and separated individuals. The proportion of widows and widowers is calculated as the share of all formerly married individuals and should be understood in the regressions as an interaction term with that variable. Being in a polygamous union is also calculated as a share of all currently married individuals and is used as an interaction term in the analysis. But the

3. A previous version of this study also included male circumcision and female genital mutilation in the regressions (de Walque 2006). Controlling for these variables does not significantly modify the coefficients on the other variables, indicating that there was no omitted variable bias due to the noninclusion of the circumcision variables. 
Table 1. Summary Statistics for Independent Variables

\begin{tabular}{|c|c|c|c|c|c|c|c|c|c|c|}
\hline \multirow[b]{2}{*}{ Variable } & \multicolumn{2}{|c|}{$\begin{array}{c}\text { Burkina Faso } \\
\quad(2003)\end{array}$} & \multicolumn{2}{|c|}{ Cameroon (2004) } & \multicolumn{2}{|c|}{ Ghana (2003) } & \multicolumn{2}{|c|}{ Kenya (2003) } & \multicolumn{2}{|c|}{$\begin{array}{c}\text { Tanzania (2003- } \\
04)\end{array}$} \\
\hline & $\begin{array}{l}\text { Men } \\
(1)\end{array}$ & $\begin{array}{l}\text { Women } \\
\text { (2) }\end{array}$ & $\begin{array}{l}\text { Men } \\
(3)\end{array}$ & $\begin{array}{l}\text { Women } \\
\text { (4) }\end{array}$ & $\begin{array}{l}\text { Men } \\
(5)\end{array}$ & $\begin{array}{l}\text { Women } \\
\text { (6) }\end{array}$ & $\begin{array}{l}\text { Men } \\
(7)\end{array}$ & $\begin{array}{l}\text { Women } \\
\text { (8) }\end{array}$ & $\begin{array}{l}\text { Men } \\
(9)\end{array}$ & $\begin{array}{l}\text { Women } \\
(10)\end{array}$ \\
\hline Urban & $\begin{array}{c}0.240 \\
(0.030)\end{array}$ & $\begin{array}{c}0.216 \\
(0.027)\end{array}$ & $\begin{array}{c}0.573 \\
(0.026)\end{array}$ & $\begin{array}{c}0.547 \\
(0.027)\end{array}$ & $\begin{array}{c}0.448 \\
(0.027)\end{array}$ & $\begin{array}{c}0.484 \\
(0.027)\end{array}$ & $\begin{array}{c}0.253 \\
(0.024)\end{array}$ & $\begin{array}{c}0.250 \\
(0.023)\end{array}$ & $\begin{array}{c}0.302 \\
(0.029)\end{array}$ & $\begin{array}{c}0.308 \\
(0.029)\end{array}$ \\
\hline Currently married & $\begin{array}{c}0.559 \\
(0.010)\end{array}$ & $\begin{array}{c}0.773 \\
(0.011)\end{array}$ & $\begin{array}{c}0.507 \\
(0.009)\end{array}$ & $\begin{array}{c}0.672 \\
(0.009)\end{array}$ & $\begin{array}{c}0.532 \\
(0.009)\end{array}$ & $\begin{array}{c}0.623 \\
(0.010)\end{array}$ & $\begin{array}{c}0.508 \\
(0.009)\end{array}$ & $\begin{array}{c}0.600 \\
(0.007)\end{array}$ & $\begin{array}{c}0.531 \\
(0.009)\end{array}$ & $\begin{array}{c}0.635 \\
(0.009)\end{array}$ \\
\hline Formerly married & $\begin{array}{c}0.018 \\
(0.003)\end{array}$ & $\begin{array}{c}0.038 \\
(0.002)\end{array}$ & $\begin{array}{c}0.091 \\
(0.004)\end{array}$ & $\begin{array}{c}0.087 \\
(0.003)\end{array}$ & $\begin{array}{c}0.060 \\
(0.004)\end{array}$ & $\begin{array}{c}0.092 \\
(0.004)\end{array}$ & $\begin{array}{c}0.041 \\
(0.004)\end{array}$ & $\begin{array}{c}0.101 \\
(0.004)\end{array}$ & $\begin{array}{c}0.054 \\
(0.003)\end{array}$ & $\begin{array}{c}0.118 \\
(0.004)\end{array}$ \\
\hline Widowed $^{\mathrm{a}}$ & $\begin{array}{c}0.172 \\
(0.061)\end{array}$ & $\begin{array}{c}0.559 \\
(0.036)\end{array}$ & n.a. & $\begin{array}{c}0.319 \\
(0.018)\end{array}$ & $\begin{array}{c}0.097 \\
(0.019)\end{array}$ & $\begin{array}{c}0.209 \\
(0.019)\end{array}$ & $\begin{array}{c}0.156 \\
(0.031)\end{array}$ & $\begin{array}{c}0.414 \\
(0.023)\end{array}$ & n.a. & n.a. \\
\hline More than one marriage & $\begin{array}{c}0.221 \\
(0.010)\end{array}$ & $\begin{array}{c}0.098 \\
(0.004)\end{array}$ & $\begin{array}{c}0.252 \\
(0.010)\end{array}$ & $\begin{array}{c}0.173 \\
(0.006)\end{array}$ & $\begin{array}{c}0.253 \\
(0.007)\end{array}$ & $\begin{array}{c}0.190 \\
(0.007)\end{array}$ & $\begin{array}{c}0.130 \\
(0.007)\end{array}$ & $\begin{array}{c}0.051 \\
(0.003)\end{array}$ & $\begin{array}{c}0.178 \\
(0.007)\end{array}$ & $\begin{array}{c}0.145 \\
(0.006)\end{array}$ \\
\hline Polygamous $^{\mathrm{a}}$ & $\begin{array}{c}0.294 \\
(0.014)\end{array}$ & $\begin{array}{c}0.483 \\
(0.012)\end{array}$ & $\begin{array}{c}0.129 \\
(0.008)\end{array}$ & $\begin{array}{c}0.305 \\
(0.011)\end{array}$ & $\begin{array}{c}0.128 \\
(0.008)\end{array}$ & $\begin{array}{c}0.227 \\
(0.012)\end{array}$ & $\begin{array}{c}0.098 \\
(0.008)\end{array}$ & $\begin{array}{c}0.186 \\
(0.009)\end{array}$ & $\begin{array}{c}0.098 \\
(0.007)\end{array}$ & $\begin{array}{c}0.097 \\
(0.008)\end{array}$ \\
\hline Education (years) & $\begin{array}{l}2.62 \\
(0.218)\end{array}$ & $\begin{array}{l}1.39 \\
(0.144)\end{array}$ & $\begin{array}{l}7.06 \\
(0.160)\end{array}$ & $\begin{array}{l}5.61 \\
(0.181)\end{array}$ & $\begin{array}{l}7.75 \\
(0.178)\end{array}$ & $\begin{array}{l}5.89 \\
(0.167)\end{array}$ & $\begin{array}{l}7.93 \\
(0.139)\end{array}$ & $\begin{array}{l}7.12 \\
(0.137)\end{array}$ & $\begin{array}{l}6.20 \\
(0.108)\end{array}$ & $\begin{array}{l}5.36 \\
(0.115)\end{array}$ \\
\hline
\end{tabular}




\begin{tabular}{lcccccccccc} 
Earth floor & 0.493 & 0.573 & 0.313 & 0.454 & 0.091 & 0.113 & 0.448 & 0.595 & 0.644 & 0.673 \\
& $(0.025)$ & $(0.025)$ & $(0.017)$ & $(0.021)$ & $(0.008)$ & $(0.010)$ & $(0.019)$ & $(0.020)$ & $(0.024)$ & $(0.024)$ \\
Muslim & 0.577 & 0.600 & 0.177 & 0.180 & 0.187 & 0.155 & 0.064 & 0.075 & 0.298 & 0.306 \\
& $(0.022)$ & $(0.020)$ & $(0.016)$ & $(0.017)$ & $(0.017)$ & $(0.015)$ & $(0.008)$ & $(0.009)$ & $(0.020)$ & $(0.020)$ \\
Catholic & 0.249 & 0.231 & 0.396 & 0.376 & 0.155 & 0.144 & 0.266 & 0.252 & 0.326 & 0.310 \\
& $(0.018)$ & $(0.014)$ & $(0.014)$ & $(0.014)$ & $(0.009)$ & $(0.009)$ & $(0.012)$ & $(0.012)$ & $(0.017)$ & $(0.016)$ \\
Protestant & 0.041 & 0.051 & 0.304 & 0.327 & 0.592 & 0.664 & 0.602 & 0.650 & 0.264 & 0.290 \\
& $(0.004)$ & $(0.005)$ & $(0.012)$ & $(0.013)$ & $(0.017)$ & $(0.017)$ & $(0.014)$ & $(0.014)$ & $(0.015)$ & $(0.016)$ \\
Other religion $^{\mathrm{b}}$ & 0.132 & 0.116 & 0.121 & 0.114 & 0.112 & 0.070 & 0.066 & 0.021 & 0.110 & 0.092 \\
& $(0.013)$ & $(0.012)$ & $(0.007)$ & $(0.010)$ & $(0.008)$ & $(0.006)$ & $(0.007)$ & $(0.003)$ & $(0.016)$ & $(0.013)$ \\
\hline
\end{tabular}

n.a. is not applicable because data were unavailable and so the variable was not included.

Note: Numbers in parentheses are clustered standard errors. The residual categories are rural and never married. The data are weighted with the sample weights given by the data provider.

${ }^{a}$ Widowed is a mean taken on formerly married individuals and polygamous is a mean taken on currently married individuals.

bIncludes animists and no religion in Burkina Faso; animists, no religion, and other religions (religions de l'éveil) in Cameroon; traditionalists and no religion in Ghana; and no religion in Kenya and Tanzania. In Ghana, other Christians are included under Protestants.

Source: Author's analysis based on data from Demographic and Health Surveys (Burkina Faso and ORC Macro 2004; Cameroon and ORC Macro 2004; Ghana and ORC Macro 2004; Kenya and ORC Macro 2004; and Tanzania and ORC Macro 2005). 
mean for the variable of having been in successive marriages (not to be confused with polygamy) is taken on the entire sample and can apply to both currently and formerly married people.

More women than men are currently married, which can be explained either by polygamy (polygyny) or by the age differences between spouses, with men generally marrying later than women. The survey includes women of ages 1549 in all surveys and men of ages 15-59 for Burkina Faso, Cameroon, and Ghana; ages 15-54 for Kenya; and ages 15-49 for Tanzania. ${ }^{4}$

Widowhood, defined as having lost one spouse and not being remarried, is not recorded in the Tanzanian survey and in the Cameroon survey only a very limited number of men are widowers. Widows and widowers constitute a substantial portion of formerly married individuals, and there are usually more widows than widowers, either because women have a longer life expectancy or because they marry older men or because it is easier for men to remarry after the death of a wife.

A large share of people have been in successive marriages, ranging from 5.1 percent of women and 13 percent of men in Kenya to 19 percent of women and 25 percent of men in Ghana. The cause of successive marriages can be either divorce or the death of the spouse. The data do not allow distinguishing between causes. Individuals whose spouse died are defined as widows or widowers if they did not remarry or as having been in successive marriages if they did remarry. The fact that more men than women have been in successive marriages suggests that it is easier for men to remarry.

There are important variations in the share of married individuals in polygamous union, ranging from 48 percent of women and 29 percent of men in Burkina Faso to 9.8 percent of men and 9.7 percent of women in Tanzania.

The proportion of individuals living in a house with an earth floor is used as a measure of poverty (table 1). The wealth quintiles provided in the Demographic and Health Surveys can be misleading as a measure of wealth, particularly for African countries. There is generally too little variation in the lowest three (sometimes four) quintiles, and differences in living standards are difficult to distinguish at those quintiles. Instead of the wealth quintiles, the analysis uses two proxies for wealth and poverty: a set of indicators for durable goods as a proxy for wealth and the presence of an earth floor as a proxy for poverty, with durable goods as instruments, letting the data pick the weights. The share of the population living in a house with an earth floor is highest in Tanzania and lowest in Ghana. Men are generally less likely to live in a house with an earth floor, but that difference is significant only in Cameroon and Kenya.

4. Regressions were also run limiting the age range for men to 15-49 to maintain parallelism with women. The results, available on request, are very similar to those for the wider age ranges for men included in this article. 
Religious affiliations have been regrouped into four categories: Muslim (the omitted dummy variable in the regressions), Catholic, Protestant, and other religions. Other religions include animists and no religion in Burkina Faso; animists, no religion, and other religions (religions de l'éveil) in Cameroon; traditionalists and no religion in Ghana; and no religion in Kenya and Tanzania. In Ghana, other Christians are included under Protestants.

\section{Methodology and Potential Sources of Bias}

Though common in the epidemiologic literature, this study does not enter sexual behaviors and other variables as controls in the HIV infection regression or in regressions with other behaviors as the dependent variable. In a crosssection analysis, the estimates derived from such regressions would suffer from reverse causality and endogeneity. For example, condom use could prevent HIV infection (expected negative association), but people who are HIV positive might be more likely to use condoms to protect their partners (reverse causality going from HIV to condom use and potentially driving a positive association). Moreover, condom use, sexual activities, and other AIDS-related practices are all choice variables that suffer from self-selection. One might, for example, expect individuals engaging in risky sexual behaviors to be more likely to use condoms because of their higher exposure.

Instead, separate regressions are run, first with HIV status as the dependent variable (tables 2 and 3 ) and then with sexual behaviors and others attitudes and practices related to HIV/AIDS epidemic as the dependent variable (table 4, and tables S3-S5 in the online supplemental appendix). Similar independent variables are entered for each set of regressions: age, location (urban and regional dummy variables), marital status, wealth, education, religion, and ethnicity. Age is included since HIV infection has a distinct hump-shaped profile, increasing until the 30-40 age range and decreasing thereafter (figures 1 and 2). Location variables account for the fact that the risk of HIV infection depends on HIV prevalence in an individual's sexual network, which is location specific. At the same time, location might determine access to prevention messages and methods such as condom use. Ethnicity and religion can also shape the sexual network and at the same time may influence the type of sexual practices considered desirable or acceptable by an individual. Marital status, including polygamy and successive marriages, is also an important determinant of the current and past sexual network. Finally, education and wealth are included as they can influence the shape of the sexual network, by conferring status and income. Education and wealth might also provide better access to prevention messages and methods and stronger incentives to avoid HIV infection. 5 The regressions with HIV as the dependent variable can be seen as the reduced form of a behavioral model in which age, location, marital

5. For an economic model linking education to incentives to avoid HIV infection, see de Walque (2007a). 
Table 2. Determinants of HIV Prevalence in Five Demographic and Health Surveys: Pooled Regressions

\begin{tabular}{|c|c|c|c|c|c|c|c|c|}
\hline \multirow[b]{2}{*}{ Variable } & \multicolumn{2}{|c|}{ All with Tanzania } & \multicolumn{2}{|c|}{ All without Tanzania } & \multicolumn{2}{|c|}{ Age 15-29 with Tanzania } & \multicolumn{2}{|c|}{ Urban with Tanzania } \\
\hline & $\begin{array}{l}\text { Men } \\
(1)\end{array}$ & $\begin{array}{l}\text { Women } \\
\text { (2) }\end{array}$ & $\begin{array}{c}\text { Men } \\
(3)\end{array}$ & $\begin{array}{l}\text { Women } \\
\text { (4) }\end{array}$ & $\begin{array}{l}\text { Men } \\
(5)\end{array}$ & $\begin{array}{l}\text { Women } \\
\text { (6) }\end{array}$ & $\begin{array}{l}\text { Men } \\
(7)\end{array}$ & $\begin{array}{l}\text { Women } \\
(8)\end{array}$ \\
\hline \multicolumn{9}{|c|}{ Linear regression model with earth floor, as a proxy for poverty, instrumented by other durable assets ${ }^{a}$} \\
\hline Years of education & $\begin{array}{r}-0.0003 \\
(0.0006)\end{array}$ & $\begin{array}{l}0.0004 \\
\quad(0.0007)\end{array}$ & $\begin{array}{r}-0.0002 \\
(0.0006)\end{array}$ & $\begin{array}{l}0 \\
\quad(0.0008)\end{array}$ & $\begin{array}{r}-0.0007 \\
(0.0006)\end{array}$ & $\begin{array}{r}-0.0008 \\
(0.0009)\end{array}$ & $\begin{array}{r}-0.0025 * * \\
\quad(0.0011)\end{array}$ & $\begin{array}{r}-0.0026 * * \\
\quad(0.0011)\end{array}$ \\
\hline Earth floor & $\begin{array}{l}0.0055 \\
\quad(0.0140)\end{array}$ & $\begin{array}{l}0.0081 \\
\quad(0.0203)\end{array}$ & $\begin{array}{l}0.0019 \\
\quad(0.0119)\end{array}$ & $\begin{array}{r}-0.0133 \\
(0.0227)\end{array}$ & $\begin{array}{l}0.0056 \\
\quad(0.0154)\end{array}$ & $\begin{aligned}- & 0.0276 \\
& (0.0245)\end{aligned}$ & $\begin{array}{l}0.0076 \\
\quad(0.0341)\end{array}$ & $\begin{array}{l}0.0436 \\
\quad(0.0383)\end{array}$ \\
\hline Urban & $\begin{array}{r}0.0309 * * * \\
(0.0081)\end{array}$ & $\begin{array}{r}0.0371 \% * * \\
(0.0098)\end{array}$ & $\begin{array}{l}0.0186 * * \\
\quad(0.0075)\end{array}$ & $\begin{array}{l}0.0237 * * \\
\quad(0.0097)\end{array}$ & $\begin{array}{r}0.0231 * * * \\
(0.0078)\end{array}$ & $\begin{array}{l}0.0182 \\
\quad(0.0118)\end{array}$ & n.a. & n.a. \\
\hline Currently married & $\begin{array}{l}0.0061 \\
\quad(0.0064)\end{array}$ & $\begin{array}{r}-0.0021 \\
(0.0073)\end{array}$ & $\begin{array}{l}0.0062 \\
\quad(0.0067)\end{array}$ & $\begin{array}{l}0.0059 \\
\quad(0.0089)\end{array}$ & $\begin{array}{l}0.0095 \\
\quad(0.0074)\end{array}$ & $\begin{array}{l}0.0097 \\
\quad(0.0077)\end{array}$ & $\begin{array}{l}0.0047 \\
\quad(0.0146)\end{array}$ & $\begin{array}{r}-0.0027 \\
(0.0120)\end{array}$ \\
\hline Formerly married & $\begin{array}{r}0.0494 \% * \% \\
(0.0130)\end{array}$ & $\begin{array}{r}0.1152 * * * \\
(0.0136)\end{array}$ & $\begin{array}{l}0.0155 \\
\quad(0.0114)\end{array}$ & $\begin{array}{r}0.0800 * * \\
(0.0195)\end{array}$ & $\begin{array}{l}0.0296^{*} \\
\quad(0.0175)\end{array}$ & $\begin{array}{r}0.1076 * * \\
(0.0192)\end{array}$ & $\begin{array}{r}0.0665 * * \\
(0.0230)\end{array}$ & $\begin{array}{r}0.1250 * \% \\
\quad(0.0221)\end{array}$ \\
\hline Widowed & n.a. & n.a. & $\begin{array}{l}0.1983 * \\
\quad(0.0883)\end{array}$ & $\begin{array}{r}0.1103 * * \\
\quad(0.0314)\end{array}$ & n.a. & n.a. & n.a. & n.a. \\
\hline More than one marriage & $\begin{array}{c}0.0188^{* * *} \\
(0.0077)\end{array}$ & $\begin{array}{r}0.0428^{* * * *} \\
(0.0076)\end{array}$ & $\begin{array}{l}0.0112 \\
\quad(0.0075)\end{array}$ & $\begin{array}{r}0.0349 \approx * \\
(0.0086)\end{array}$ & $\begin{array}{l}0.0084 \\
\quad(0.0154)\end{array}$ & $\begin{array}{r}0.0572 * * * * \\
(0.0142)\end{array}$ & $\begin{array}{l}0.0139 \\
(0.0136)\end{array}$ & $\begin{array}{r}0.0516 * * * \\
(0.0141)\end{array}$ \\
\hline Polygamous & $\begin{array}{r}-0.0095 \\
(0.0106)\end{array}$ & $\begin{array}{l}0.0156 * * \\
(0.0065)\end{array}$ & $\begin{array}{r}-0.007 \\
(0.0111)\end{array}$ & $\begin{array}{l}0.0167 * * \\
(0.0071)\end{array}$ & $\begin{array}{r}-0.0075 \\
(0.0276)\end{array}$ & $\begin{array}{l}0.0287 * * \\
\quad(0.0117)\end{array}$ & $\begin{array}{l}0.0157 \\
\quad(0.0288)\end{array}$ & $\begin{array}{l}0.0214 \\
\quad(0.0163)\end{array}$ \\
\hline Protestant & $\begin{aligned}- & 0.0022 \\
& (0.0051)\end{aligned}$ & $\begin{array}{r}-0.0064 \\
(0.0058)\end{array}$ & $\begin{array}{l}0.0033 \\
\quad(0.0052)\end{array}$ & $\begin{array}{r}-0.0033 \\
(0.0065)\end{array}$ & $\begin{array}{l}0.0012 \\
\quad(0.0059)\end{array}$ & $\begin{array}{r}-0.0017 \\
(0.0073)\end{array}$ & $\begin{array}{l}-0.01 \\
\quad(0.0094)\end{array}$ & $\begin{array}{r}-0.0212 * \\
\quad(0.0117)\end{array}$ \\
\hline Muslim & $\begin{array}{r}-0.0011 \\
(0.0061)\end{array}$ & $\begin{array}{r}-0.0053 \\
(0.0069)\end{array}$ & $\begin{array}{l}0.0017 \\
\quad(0.0056)\end{array}$ & $\begin{array}{r}-0.0133^{*} \\
(0.0065)\end{array}$ & $\begin{array}{l}0.0026 \\
(0.0069)\end{array}$ & $\begin{array}{r}-0.0023 \\
(0.0079)\end{array}$ & $\begin{array}{r}-0.0067 \\
(0.0121)\end{array}$ & $\begin{array}{r}-0.0234 * \\
(0.0139)\end{array}$ \\
\hline
\end{tabular}




\begin{tabular}{|c|c|c|c|c|c|c|c|c|}
\hline Other religion & $\begin{array}{r}-0.0087 \\
(0.0069)\end{array}$ & $\begin{array}{c}-0.0238 * * \\
(0.0097)\end{array}$ & $\begin{array}{l}0.0033 \\
\quad(0.0072)\end{array}$ & $\begin{array}{r}-0.0177^{*} \\
(0.0099)\end{array}$ & $\begin{array}{r}-0.0129 * \\
(0.0069)\end{array}$ & $\begin{array}{c}-0.0207 \\
(0.0129)\end{array}$ & $\begin{array}{r}-0.0193 \\
(0.0158)\end{array}$ & $\begin{array}{c}-0.0448 * \\
(0.0220)\end{array}$ \\
\hline Observations & 19,986 & 23,085 & 15,209 & 17,408 & 11,007 & 13,486 & 6,602 & 7,893 \\
\hline$R$-square & 0.05 & 0.07 & 0.05 & 0.08 & 0.04 & 0.07 & 0.08 & 0.09 \\
\hline \multicolumn{9}{|c|}{ Marginal effects of probit estimations, with durable asset dummy variables controlling for wealth ${ }^{\mathrm{b}}$ (not shown) } \\
\hline Years of education & $\begin{array}{r}-0.0003 \\
(0.0004)\end{array}$ & $\begin{array}{c}-0.0002 \\
(0.0004)\end{array}$ & $\begin{array}{r}-0.0002 \\
(0.0004)\end{array}$ & $\begin{array}{r}-0.0003 \\
(0.0005)\end{array}$ & $\begin{array}{r}-0.0005 \\
(0.0003)\end{array}$ & $\begin{array}{r}-0.0008 * \\
(0.0005)\end{array}$ & $\begin{array}{c}-0.0012 * * \\
(0.0006)\end{array}$ & $\begin{array}{c}-0.0027 * * * \\
(0.0008)\end{array}$ \\
\hline Assets $(2(10)$-Test & 31.66 & 41.34 & 12.76 & 15.50 & 8.51 & 29.19 & 18.13 & 14.86 \\
\hline Prob $>\chi^{2}$ & 0.0005 & 0.0000 & 0.2377 & 0.1149 & 0.5791 & 0.0012 & 0.0528 & 0.1372 \\
\hline
\end{tabular}

*Significant at the 10 percent level.

$*$ Significant at the 5 percent level.

$* *$ Significant at the 1 percent level.

n.a. is not applicable because ethnicity and widowhood data were unavailable for Tanzania and urban location is not relevant for regressions 7 and 8 focusing on the urban sample. Regressions 1-4 were therefore run with and without Tanzania.

Note: Numbers in parentheses are robust and clustered standard errors. HIV prevalence is the dependent variable. Data from the five surveys have been pooled. Dummy variables controlling for age, country and region, and ethnicity (regressions 3 and 4) are also included. The omitted dummy variables are rural, never married, and Catholic (see note in table 1). The data are weighted with the sample weights given by the data provider, multiplied by the country population divided by the sample size.

aTo control for wealth/poverty, earth floor is included in the linear regression specifications and instrumented by the other durable assets: type of latrine, type of floor (earth floor or not), electricity, refrigerator, radio, television, bicycle, motorcycle, or car.

${ }^{\mathrm{b}}$ All asset dummy variables are included (coefficients not shown, but the results of an F-test of joint significance are reported).

Source: Author's analysis based on data from Demographic and Health Surveys (Burkina Faso and ORC Macro 2004; Cameroon and ORC Macro 2004; Ghana and ORC Macro 2004; Kenya and ORC Macro 2004; and Tanzania and ORC Macro 2005). 
Table 3. Determinants of HIV Prevalence in Five Demographic and Health Surveys: Analysis by Country

\begin{tabular}{|c|c|c|c|c|c|c|c|c|c|c|}
\hline \multirow[b]{2}{*}{ Variable } & \multicolumn{2}{|c|}{ Burkina Faso (2003) } & \multicolumn{2}{|c|}{ Cameroon (2004) } & \multicolumn{2}{|c|}{ Ghana (2003) } & \multicolumn{2}{|c|}{ Kenya (2003) } & \multicolumn{2}{|c|}{ Tanzania 2004} \\
\hline & $\begin{array}{l}\text { Men } \\
(1)\end{array}$ & $\begin{array}{l}\text { Women } \\
(2)\end{array}$ & $\begin{array}{l}\text { Men } \\
(3)\end{array}$ & $\begin{array}{l}\text { Women } \\
\text { (4) }\end{array}$ & $\begin{array}{c}\text { Men } \\
(5)\end{array}$ & $\begin{array}{l}\text { Women } \\
\text { (6) }\end{array}$ & $\begin{array}{c}\text { Men } \\
(7)\end{array}$ & $\begin{array}{l}\text { Women } \\
(8)\end{array}$ & $\begin{array}{l}\text { Men } \\
(9)\end{array}$ & $\begin{array}{l}\text { Women } \\
(10)\end{array}$ \\
\hline \multicolumn{11}{|c|}{ Linear regression model with earth floor, as proxy for poverty, instrumented by the other durable assets ${ }^{\mathrm{a}}$} \\
\hline $\begin{array}{l}\text { Years of } \\
\text { education }\end{array}$ & $\begin{array}{l}0.0006 \\
(0.0014)\end{array}$ & $\begin{array}{r}-0.0023 \\
(0.0014)\end{array}$ & $\begin{array}{l}-0.0004 \\
(0.0010)\end{array}$ & $\begin{array}{c}-0.0009 \\
(0.0018)\end{array}$ & $\begin{array}{r}0.0001 \\
(0.0006)\end{array}$ & $\begin{array}{l}0.0004 \\
(0.0008)\end{array}$ & $\begin{array}{c}-0.0002 \\
(0.0015)\end{array}$ & $\begin{array}{r}-0.0021 \\
(0.0020)\end{array}$ & $\begin{array}{r}-0.0006 \\
(0.0017)\end{array}$ & $\begin{array}{l}0.0009 \\
(0.0017)\end{array}$ \\
\hline Earth floor & $\begin{array}{l}0.0065 \\
(0.0171)\end{array}$ & $\begin{array}{r}-0.0112 \\
(0.0249)\end{array}$ & $\begin{array}{l}-0.0186 \\
(0.0125)\end{array}$ & $\begin{array}{l}-0.009 \\
(0.0291)\end{array}$ & $\begin{array}{r}0.0072 \\
(0.0272)\end{array}$ & $\begin{array}{l}0.045 \\
(0.0491)\end{array}$ & $\begin{array}{c}0.0223 \\
(0.0169)\end{array}$ & $\begin{array}{r}-0.0472 \\
(0.0387)\end{array}$ & $\begin{array}{l}0.015 \\
(0.0431)\end{array}$ & $\begin{array}{l}0.0192 \\
(0.0360)\end{array}$ \\
\hline Urban & $\begin{array}{l}0.016 \\
(0.0131)\end{array}$ & $\begin{array}{r}0.0156 \\
(0.0157)\end{array}$ & $\begin{array}{r}0.0141 \\
(0.0086)\end{array}$ & $\begin{array}{l}0.0332 * * \\
(0.0152)\end{array}$ & $\begin{array}{r}0.0021 \\
(0.0048)\end{array}$ & $\begin{array}{l}0.0104 \\
(0.0082)\end{array}$ & $\begin{array}{l}0.0326^{*} \\
(0.0193)\end{array}$ & $\begin{array}{l}0.018 \\
(0.0225)\end{array}$ & $\begin{array}{l}0.0603 * * \\
(0.0259)\end{array}$ & $\begin{array}{l}0.0592^{* * *} \\
(0.0228)\end{array}$ \\
\hline $\begin{array}{l}\text { Currently } \\
\text { married }\end{array}$ & $\begin{array}{l}0.0276 * * * \\
(0.0096)\end{array}$ & $\begin{array}{r}-0.0154 \\
(0.0109)\end{array}$ & $\begin{array}{l}-0.0076 \\
(0.0109)\end{array}$ & $\begin{array}{l}0.0094 \\
(0.0133)\end{array}$ & $\begin{array}{c}0.002 \\
(0.0064)\end{array}$ & $\begin{array}{r}-0.0059 \\
(0.0089)\end{array}$ & $\begin{array}{c}0.0013 \\
(0.0150)\end{array}$ & $\begin{array}{l}0.0033 \\
(0.0172)\end{array}$ & $\begin{array}{l}0.0156 \\
(0.0136)\end{array}$ & $\begin{array}{c}-0.0082 \\
(0.0129)\end{array}$ \\
\hline Formerly married & $\begin{array}{l}0.025 \\
(0.0229)\end{array}$ & $\begin{array}{r}-0.0009 \\
(0.0279)\end{array}$ & $\begin{array}{r}0.0109 \\
(0.0140)\end{array}$ & $\begin{array}{l}0.0919 * * * \\
(0.0248)\end{array}$ & $\begin{array}{r}0.0109 \\
(0.0118)\end{array}$ & $\begin{array}{l}0.0310 * \\
(0.0159)\end{array}$ & $\begin{array}{c}0.0067 \\
(0.0275)\end{array}$ & $\begin{array}{l}0.1028 * * * \\
(0.0388)\end{array}$ & $\begin{array}{l}0.0900 * * * \\
(0.0295)\end{array}$ & $\begin{array}{l}0.1228 * * \\
(0.0205)\end{array}$ \\
\hline Widowed & $\begin{array}{l}0.0867 \\
(0.1024)\end{array}$ & $\begin{array}{r}0.0296 \\
(0.0402)\end{array}$ & $\begin{array}{c}0.0 \\
(0.0000)\end{array}$ & $\begin{array}{l}0.1302 * * * \\
(0.0451)\end{array}$ & $\begin{array}{l}-0.0224 \\
(0.0271)\end{array}$ & $\begin{array}{l}0.0056 \\
(0.0290)\end{array}$ & $\begin{array}{l}0.3070 * * \\
(0.1287)\end{array}$ & $\begin{array}{l}0.1396 * * \\
(0.0556)\end{array}$ & n.a. & n.a. \\
\hline $\begin{array}{l}\text { More than one } \\
\text { marriage }\end{array}$ & $\begin{array}{l}0.0088 \\
(0.0138)\end{array}$ & $\begin{array}{r}0.0155 \\
(0.0104)\end{array}$ & $\begin{array}{l}-0.0004 \\
(0.0094)\end{array}$ & $\begin{array}{l}0.0405 * * * \\
(0.0111)\end{array}$ & $\begin{array}{r}0.0137 \\
(0.0085)\end{array}$ & $\begin{array}{l}0.0288 * * * \\
(0.0090)\end{array}$ & $\begin{array}{c}0.0122 \\
(0.0216)\end{array}$ & $\begin{array}{l}0.0563 \\
(0.0346)\end{array}$ & $\begin{array}{l}0.0346 * * \\
(0.0174)\end{array}$ & $\begin{array}{l}0.0600 * *: \\
(0.0168)\end{array}$ \\
\hline Polygamous & $\begin{array}{r}-0.0214 \% \\
(0.0130)\end{array}$ & $\begin{array}{r}-0.0091 \\
(0.0059)\end{array}$ & $\begin{array}{l}-0.0023 \\
(0.0129)\end{array}$ & $\begin{array}{l}0.0045 \\
(0.0109)\end{array}$ & $\begin{array}{l}-0.0175 \\
(0.0136)\end{array}$ & $\begin{array}{l}0.0137 \\
(0.0101)\end{array}$ & $\begin{array}{c}0.0127 \\
(0.0352)\end{array}$ & $\begin{array}{l}0.0364 * \\
(0.0195)\end{array}$ & $\begin{array}{r}-0.0033 \\
(0.0254)\end{array}$ & $\begin{array}{l}0.0216 \\
(0.0162)\end{array}$ \\
\hline Protestant & $\begin{array}{l}0.0278 \\
(0.0235)\end{array}$ & $\begin{array}{r}-0.0002 \\
(0.0111)\end{array}$ & $\begin{array}{l}-0.0011 \\
(0.0074)\end{array}$ & $\begin{array}{r}-0.0068 \\
(0.0096)\end{array}$ & $\begin{array}{r}0.0075 \\
(0.0055)\end{array}$ & $\begin{array}{r}-0.0097 \\
(0.0079)\end{array}$ & $\begin{array}{c}0.0028 \\
(0.0097)\end{array}$ & $\begin{array}{r}-0.0009 \\
(0.0115)\end{array}$ & $\begin{array}{r}-0.0146 \\
(0.0108)\end{array}$ & $\begin{array}{r}-0.0144 \\
(0.0120)\end{array}$ \\
\hline Muslim & $\begin{array}{l}0.0119 \\
(0.0079)\end{array}$ & $\begin{array}{r}-0.0006 \\
(0.0076)\end{array}$ & $\begin{array}{r}0.0114 \\
(0.0119)\end{array}$ & $\begin{array}{r}-0.005 \\
(0.0169)\end{array}$ & $\begin{array}{r}0.0008 \\
(0.0074)\end{array}$ & $\begin{array}{r}-0.0006 \\
(0.0098)\end{array}$ & $\begin{array}{r}-0.0072 \\
(0.0228)\end{array}$ & $\begin{array}{r}-0.0521 * \\
(0.0289)\end{array}$ & $\begin{array}{r}-0.0136 \\
(0.0119)\end{array}$ & $\begin{array}{l}0.001 \\
(0.0140)\end{array}$ \\
\hline
\end{tabular}




\begin{tabular}{|c|c|c|c|c|c|c|c|c|c|c|}
\hline Other religion & $\begin{array}{l}0.005 \\
(0.0106)\end{array}$ & $\begin{array}{r}-0.0102 \\
(0.0069)\end{array}$ & $\begin{array}{l}-0.0109 \\
(0.0084)\end{array}$ & $\begin{array}{c}-0.0256 * * \\
(0.0116)\end{array}$ & $\begin{array}{r}0.0102 \\
(0.0134)\end{array}$ & $\begin{array}{r}-0.0204 \\
(0.0124)\end{array}$ & $\begin{array}{c}0.0204 \\
(0.0175)\end{array}$ & $\begin{array}{l}0.0106 \\
(0.0514)\end{array}$ & $\begin{array}{c}-0.0323 * * \\
(0.0149)\end{array}$ & $\begin{array}{r}-0.0369 * \\
(0.0196)\end{array}$ \\
\hline Observations & 3339 & 4164 & 4997 & 5085 & 3959 & 4919 & 2914 & 3240 & 4772 & 5665 \\
\hline$R$-square & 0.06 & 0.04 & 0.06 & 0.1 & 0.04 & 0.03 & 0.11 & 0.12 & 0.05 & 0.08 \\
\hline \multicolumn{11}{|c|}{ Marginal effects of probit estimations, with durable asset dummies controlling for wealth (not shown) ${ }^{\mathrm{b}}$} \\
\hline $\begin{array}{l}\text { Years of } \\
\text { education }\end{array}$ & $\begin{array}{l}0 \\
(0.0002)\end{array}$ & $\begin{array}{c}-0.0006^{*} \\
(0.0003)\end{array}$ & $\begin{array}{l}-0.0003 \\
(0.0008)\end{array}$ & $\begin{array}{r}-0.0012 \\
(0.0010)\end{array}$ & $\begin{array}{c}0 \\
(0.0002)\end{array}$ & $\begin{array}{l}0.0002 \\
(0.0005)\end{array}$ & $\begin{array}{l}0 \\
(0.0009)\end{array}$ & $\begin{array}{c}-0.0008 \\
(0.0013)\end{array}$ & $\begin{array}{c}-0.0009 \\
(0.0012)\end{array}$ & $\begin{array}{l}0 \\
(0.0011)\end{array}$ \\
\hline $\begin{array}{l}\text { Assets } \chi^{2} \\
(10) \text {-Test }\end{array}$ & 28.07 & 18.92 & 17.29 & 14.67 & 21.29 & 11.92 & 11.79 & 9.63 & 26.15 & 42.88 \\
\hline \multicolumn{11}{|c|}{ Mean HIV prevalence } \\
\hline Mean HIV & $\begin{array}{l}0.0194 \\
(0.0031)\end{array}$ & $\begin{array}{r}0.0182 \\
(0.0027)\end{array}$ & $\begin{array}{r}0.0391 \\
(0.0030)\end{array}$ & $\begin{array}{l}0.0662 \\
(0.0043)\end{array}$ & $\begin{array}{r}0.0162 \\
(0.0022)\end{array}$ & $\begin{array}{l}0.0270 \\
(0.0024)\end{array}$ & $\begin{array}{c}0.0463 \\
(0.0051)\end{array}$ & $\begin{array}{l}0.0868 \\
(0.0064)\end{array}$ & $\begin{array}{l}0.0626 \\
(0.0047)\end{array}$ & $\begin{array}{l}0.0769 \\
(0.0052)\end{array}$ \\
\hline
\end{tabular}

Significant at the *10 percent level.

$*$ :Significant at the 5 percent level.

$* *$ Significant at the 1 percent level.

n.a. is not applicable because data were unavailable and so the variable was not included.

Note: Numbers in parentheses are robust and clustered standard errors. HIV prevalence is the dependent variable. Controls for age, region, and ethnicity are also included; ethnicity and widowhood are not controlled for in Tanzania 2004 as the variable were not available. The omitted dummy variables are rural, never married, and Muslim (see note in table 1). The data are weighted with the sample weights given by the data provider.

${ }^{\text {a }}$ To control for wealth/poverty, earth floor is included in the linear regression specifications and instrumented by the other durable assets: type of latrine, type of floor (earth floor or not), electricity, refrigerator, radio, television, bicycle, motorcycle, or car.

${ }^{\mathrm{b}}$ All asset dummy variables are included (coefficients not shown, but the results of an F-test of joint significance are reported).

Source: Author's analysis based on data from Demographic and Health Surveys (Burkina Faso and ORC Macro 2004; Cameroon and ORC Macro 2004; Ghana and ORC Macro 2004; Kenya and ORC Macro 2004; and Tanzania and ORC Macro 2005). 


\begin{tabular}{|c|c|c|c|c|c|c|}
\hline \multirow[b]{2}{*}{ Variable } & \multicolumn{2}{|c|}{ All with Tanzania } & \multicolumn{2}{|c|}{ All without Tanzania } & \multicolumn{2}{|c|}{ Age 15-29 with Tanzania } \\
\hline & $\begin{array}{c}\text { Men } \\
(1)\end{array}$ & $\begin{array}{c}\text { Women } \\
(2)\end{array}$ & $\begin{array}{c}\text { Men } \\
(3)\end{array}$ & $\begin{array}{c}\text { Women } \\
(4)\end{array}$ & $\begin{array}{c}\text { Men } \\
(5)\end{array}$ & $\begin{array}{c}\text { Women } \\
(6)\end{array}$ \\
\hline \multicolumn{7}{|c|}{ Determinants of using a condom at the last intercourse with spouse (married sample) } \\
\hline Years of education & $0.0026 * *$ & $0.0034 * *$ & $0.0027 * * *$ & $0.0039 * *$ & $0.0076 * *$ & $0.0041 * * *$ \\
\hline Linear & $(0.0009)$ & $(0.0006)$ & $(0.0009)$ & $(0.0007)$ & $(0.0020)$ & $(0.0010)$ \\
\hline Observations & 10440 & 23883 & 7671 & 19895 & 2474 & 11524 \\
\hline Years of education & $0.0031 * *$ & $0.0026 * *$ & $0.0028 * *$ & $0.0026 * * *$ & $0.0071 * *$ & $0.0029 * * *$ \\
\hline Probit, marginal effects & $(0.0006)$ & $(0.0003)$ & $(0.0006)$ & $(0.0003)$ & $(0.0017)$ & $(0.0006)$ \\
\hline \multicolumn{7}{|c|}{ Determinants of using a condom at the last intercourse if not with spouse (if nonmarital sex) } \\
\hline Years of education & $0.0188 * * *$ & $0.0149 * * *$ & $0.0170 * * *$ & $0.0131 * * *$ & $0.0176 * * *$ & $0.0182 * * *$ \\
\hline Linear & $(0.0029)$ & $(0.0029)$ & $(0.0033)$ & $(0.0030)$ & $(0.0037)$ & $(0.0036)$ \\
\hline Observations & 5530 & 5454 & 4177 & 4485 & 4303 & 4219 \\
\hline$R$-square & 0.14 & 0.13 & 0.14 & 0.1 & 0.14 & 0.12 \\
\hline Years of education & $0.0215 * *$ & $0.0224 * * *$ & $0.0202 * * *$ & $0.0212 * * *$ & $0.0236 * * *$ & $0.0278 * * *$ \\
\hline \multicolumn{7}{|c|}{ Determinants of having nonmarital sex in the last 12 months (currently married) } \\
\hline Years of education & 0.0008 & $0.0028 * * *$ & 0.0001 & $0.0033 * * *$ & -0.0059 & $0.0052 * * *$ \\
\hline Linear & $(0.0013)$ & $(0.0005)$ & $(0.0013)$ & $(0.0005)$ & $(0.0037)$ & $(0.0009)$ \\
\hline Observations & 11980 & 28736 & 8959 & 24518 & 3021 & 13839 \\
\hline$R$-square & 0.12 & 0.11 & 0.15 & 0.13 & 0.13 & 0.15 \\
\hline Years of education & $0.0036 * *$ & $0.0012 * *$ & $0.0024 * *$ & $0.0013 * * *$ & -0.0019 & $0.0023 * * *$ \\
\hline Probit, marginal effects & $(0.0012)$ & $(0.0002)$ & $(0.0012)$ & $(0.0002)$ & $(0.0035)$ & $(0.0004)$ \\
\hline
\end{tabular}

Significant at the $* 10$ percent level.

* Significant at the 5 percent level.

$* *$ Significant at the 1 percent level.

Note: Numbers in parentheses are robust and clustered standard errors. Controls for age (dummy variables), urban location, marital status, religion, region, and ethnicity are also included (ethnicity and widowhood are not controlled for in Tanzania 2004 as the variable were not available). To control for wealth/poverty, earth floor (coefficient not shown) is included and instrumented by the other during assets: type of latrine, type of floor (earth floor or not), electricity, refrigerator, radio, television, bicycle, motorcycle, or car. In the probit specification, all asset dummy variables are included (coefficients not shown). The data are weighted with the sample weights given by the data provider, multiplied by the country population divided by the sample size. 
Figure 1. Age Profile of HIV Prevalence in Men in Five African Countries

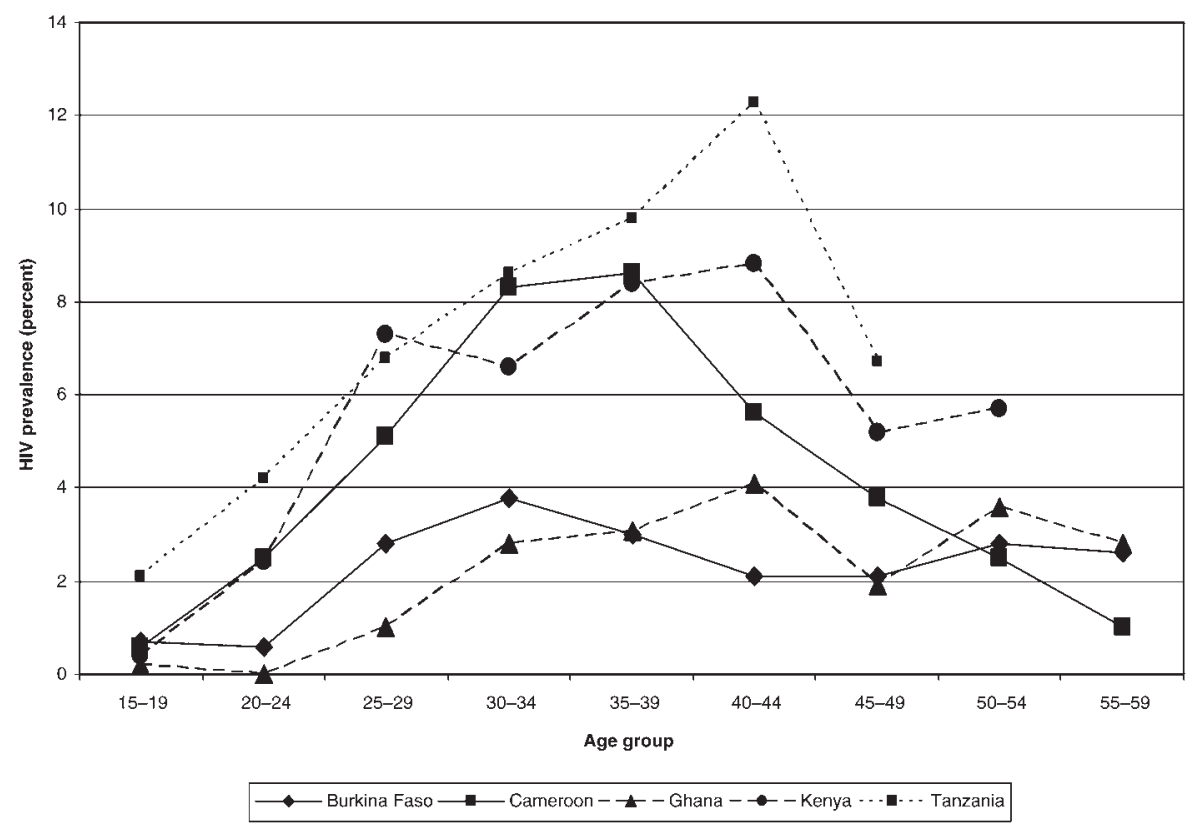

Source: Author's analysis based on data from Demographic and Health Surveys (Burkina Faso and ORC Macro 2004; Cameroon and ORC Macro 2004; Ghana and ORC Macro 2004; Kenya and ORC Macro 2004; and Tanzania and ORC Macro 2005).

status, religion, ethnicity, education, and wealth influence the composition of the sexual network, sexual practices, and prevention measures, which in turn can have an impact on the risk of HIV infection.

Most of the individual characteristics used as regressors, with the exception of age and ethnic background, cannot be defined as completely exogenous variables. Location, marital status, education, wealth, and even religion are, at least to some extent, choice variables for individuals or their family. The data set does not offer sources of exogenous variations for those variables. Coefficients should therefore be interpreted with caution and as associations rather than causal effects. In particular, marital status and marital history are clearly endogenous variables, and their inclusion as regressors could be questioned. For example, the positive associations between HIV and widowhood and successive marriages are very likely to be influenced by reverse causality. As a robustness check, a specification is proposed that excludes marital status and marital history (table S2, model 2, in the supplemental appendix). The main result of the analysis-the absence of a gradient between education and HIV infection-does not change. ${ }^{6}$

6. There is one negative coefficient, for Kenyan women, which is statistically significant at the 10 percent level. 
Figure 2. Age Profile of HIV Prevalence in Women in Five African Countries

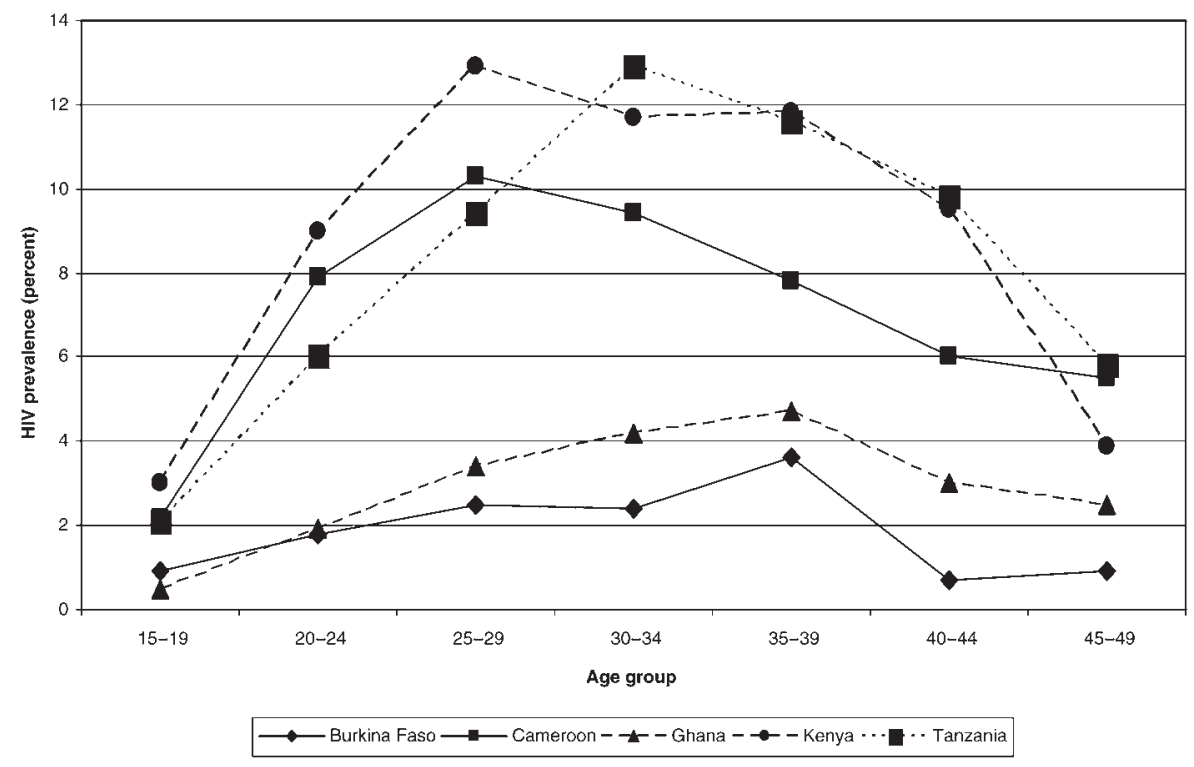

Source: Author's analysis based on data from Demographic and Health Surveys (Burkina Faso and ORC Macro 2004; Cameroon and ORC Macro 2004; Ghana and ORC Macro 2004; Kenya and ORC Macro 2004; and Tanzania and ORC Macro 2005).

Sexual behavior and other practices are all self-reported. This is an obvious but inescapable limitation. Diverging reports on self-reported behaviors between husbands and wives (for example more married men report using a condom in marriage or discussing AIDS with their spouse than do married women) lead to the suspicion that some behaviors are not truthfully reported. Gersovitz (2005) discusses the issue of self-reporting sexual behaviors in the Demographic and Health Surveys and shows several inconsistencies, in particular regarding virginity and the age at first sexual intercourse. Some discrepancies in reported sexual behavior between men and women in condom use and number of partners, for example, can potentially be explained by the fact that the extramarital partners of men with a high intensity of sexual activity, typically commercial sex workers, are not included or are underrepresented in the survey. De Walque (2007b), however, shows that the reports of men and women in couples are not always mutually consistent, even when, in theory, they should be, as in the case of condom use during the last sexual intercourse between the two interviewed partners. Polygamy, which is frequent in the studied countries, might also explain some of the reported discrepancies between married men and women. The self-reported feature of the sexual behavior variable would bias the analysis, especially if the reporting bias varies with education. For example if because of perceived social desirability more educated individuals are more likely to report that they are using condoms-even if 
they are not-this would create a bias toward a positive association between condom use and education.

One dependent variable that is not self-reported is HIV status, which is determined by an HIV test on a blood sample. Including HIV testing is one of the great advantages of the new Demographic and Health Surveys. However, some individuals who had been assigned to the sample for HIV testing refused to be tested or were absent. If the absence of a test is not random, this could be a source of bias. Table S1 deals with this issue and shows that acceptance of the test is somewhat less likely in urban areas. However, coverage of the HIV test is usually high (at $82-95$ percent).

Antiretroviral treatment is currently scaled-up in the five survey countries. Although the data do not allow analysis of that issue (though table S5a indicates that educated and, to a lesser extent, richer people are more likely to use voluntary counseling and testing services), access to treatment is expected to be easier in urban centers and for educated and richer people. If access to treatment keeps those people alive while its absence implies that poorer and less educated individuals in rural areas are more likely to die, this would bias upward the coefficients on education, wealth, and urban location in a regression with HIV status as the dependent variable. This should be kept in mind in the analysis, even if only a small share of HIV positive individuals is receiving treatment.

Indeed, only a small percentage of the HIV positive population is medically eligible for treatment. There is a long interval between HIV infectionseroconversion-and development of AIDS. For adults in Uganda, the median time from seroconversion to AIDS has been estimated at 9.4 years (Morgan and others 2002). Antiretroviral treatment is recommended only for individuals at the AIDS stage (generally, with fewer than 200 CD4 cells per cubic millimeter). In addition, access to treatment programs is recent and available to only a small percentage of medically eligible patients. Estimates of the proportion of people who are HIV positive and are receiving treatment varied from 0.66 percent in Tanzania to 5.35 percent in Burkina Faso. ${ }^{7}$ The number of people on treatment was probably even smaller in 2003 and 2004, when the data for this study were collected.

The regressions use the sample weights provided in the Demographic and Health Surveys, ${ }^{8}$ and the standard errors are clustered at the enumeration-area level.

Tables 3 and S1-S5 present results with country-specific regressions. However, since the Demographic and Health Surveys are defined similarly in each country, it is also interesting to pool the data from the five countries in a single regression, increasing the power of the analysis. The results of pooled

7. The estimates are 4 percent for Cameroon and Kenya and 1.3 percent for Ghana. These figures are calculated by the author and are based on data on treatment coverage from June 2005 (WHO and UNAIDS 2005 and Burkina Faso Government 2006).

8. Table S2 presents as a robustness check of results from unweighted regressions and shows very similar results. 
regressions are included in table 2 (for HIV status) and table 4 (for sexual behavior). One condition for pooling the data across surveys is that the coefficients on the interaction between a country indicator and the variable of interest not be significantly different across countries. In the pooled regressions, the data are weighted by the sample weight provided in the survey, multiplied by the country population and divided by the sample size for each survey.

\section{HIV STATUS}

For both genders and in almost all countries, the age profile for HIV prevalence is hump shaped, first increasing with age and then decreasing (figures 1 and 2). The peak of HIV prevalence is at older ages in the countries with low overall HIV prevalence, such as Burkina Faso and Ghana. That peak is generally earlier for women than for men, with the exception of Burkina Faso. This is explained by women's tendency to initiate their sexual activity earlier than men do (table S4c, with an exception in Kenya) and by the higher estimated biologic probability of transmission from men to women than from women to men. The age profile seems to be more tilted toward older ages (to the right) in Tanzania and Ghana and for women in Burkina Faso while it is more tilted toward younger ages (to the left) in Cameroon and Kenya and for men in Burkina Faso.

HIV prevalence is not a perfect measure of the current state of the epidemic since it is a stock variable, affected by past incidence and mortality rates. As noted, individuals who are HIV positive are asymptomatic for nine years on average before they get AIDS. Without treatment, individuals with AIDS die within one year on average. Therefore, lower HIV prevalence at older ages does not necessary mean that those birth cohorts were less likely to be infected but rather that a substantial portion of individuals in those birth cohorts who were HIV positive have already died.

As a starting point for the analysis and for comparison with regression coefficients in multivariate analyses, table 5 reports unadjusted means of HIV prevalence by education and by wealth and poverty levels from reports of the Demographic and Health Surveys (Burkina Faso Government and ORC Macro 2004; Cameroon Government and ORC Macro 2004; Ghana Government and ORC Macro 2004; Kenya Government and ORC Macro 2004; and Tanzania Government and ORC Macro 2005). These unadjusted means suggest that HIV infection generally increases with educational attainment, although the increase seems larger and more consistent from no education to some primary education than from some primary education to some secondary education or more. The unadjusted means also suggest that the risk of HIV infection increases with wealth. In every country, individuals living in a household with an earth floor (the proxy for poverty) are less likely to be HIV positive than those having another type of floor, who are considered less poor. That difference, however, is statistically significant at the 5 percent level only in Cameroon and Tanzania, for both men and women, and at the 10 percent 
Table 5. HIV Prevalence by Selected Characteristics: Unadjusted Means

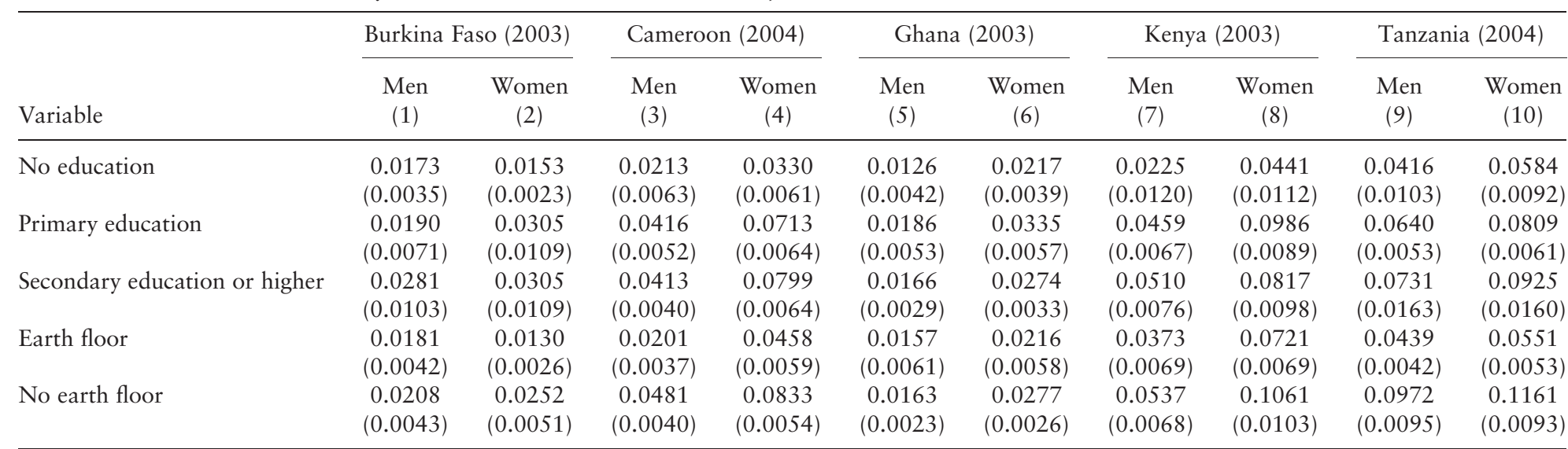

Note: Numbers in parentheses are clustered standard errors. The data are weighted with the sample weights given by the data provider.

Source: Author's analysis based on data from Demographic and Health Surveys (Burkina Faso and ORC Macro 2004; Cameroon and ORC Macro 2004; Ghana and ORC Macro 2004; Kenya and ORC Macro 2004; and Tanzania and ORC Macro 2005). 
confidence level for women in Kenya. The analysis in the remainder of this article goes beyond unadjusted means.

The next analysis pools the observations for the five countries in regressions in which the dependent variable is HIV status (zero for HIV negative and one for HIV positive; table 2). The analysis controls for wealth using a group of assets as indicators of wealth rather than asset quintiles, which are not easily compared across countries. These assets are type of latrine, type of floor, availability of electricity, and ownership of a refrigerator, radio, television, bicycle, motorcycle, or car. For comparison with the coefficients from the linear model, results are also shown for a probit specification for cases where the dependent variable is binary (displaying the marginal effects of the probit coefficient). Two alternative methods are used to control for wealth or poverty. The linear models include the presence of an earth floor as a proxy for poverty and use the other durable goods as instruments, letting the data pick the weights. The probit specifications use a set of indicators for durable goods as a proxy for wealth.

Because the ethnicity ${ }^{9}$ and the widowhood variables were not available in the Tanzania survey, results are presented in table 2 with Tanzania and without (regressions 3 and 4). Table 2 also includes results for the entire age range (regressions $1-4,7$, and 8 ) and, because a previous study documented a negative relationship between education and HIV infection among women younger than 30 in rural Uganda (de Walque, 2007a), ${ }^{10}$ for the age group $15-$ 29 (columns 5 and 6).

Education does not seem to be significantly associated with HIV infection. ${ }^{11}$ However, in regressions 7 and 8, which restrict the sample to urban areas, coefficients on years of education are negative and statistically significant. ${ }^{12}$ This finding suggests that while it may be difficult to isolate a negative relationship between education and HIV in the overall population, it can be measured in urban settings. It may be that the negative relationship between education

9. In the pooled regressions without Tanzania, the ethnicity indicators are interacted with the country indicators so that each country maintains its own set of ethnicity categories.

10. This was one of the first studies to show a negative gradient. Of 27 studies reviewed by Hargreaves and Glynn (2002), only one, on sugar estate workers in Ethiopia, reported a significantly negative association between HIV infection and education. Most of these studies, however, are in urban settings and based on data collected in the beginning of the 1990s, at an earlier stage of the epidemic.

11. An exception is among young women of ages $15-29$, for whom more education is negatively associated with HIV infection (at the 10 percent significance level) under the probit specification, but not in the linear instrumental variables regression. The sample was also restricted to individuals under age 30 in the country-level regressions to determine whether the association between education and HIV status was different for younger individuals: only in the case of young women in Kenya is there a significant negative association between education and the risk of HIV infection (results available on request).

12. Regressions were also separated for the urban and rural samples in the country-level regressions, but no consistent or significant pattern was found (results available on request). This might due to the fact that the urban samples are rather small in each individual Demographic and Health Survey, increasing the interest in using pooled regressions. 
and HIV infection takes time to develop and is found earlier in cities, where information spreads faster and HIV prevalence is generally higher.

Urban location tends to be positively associated with HIV infection, as does being formerly married. Widowed individuals are even more at risk (table 2, regressions 3 and 4). It is likely that marital disruption and widowhood are a consequence rather than a cause of HIV infection and that widowed individuals are more likely to be HIV positive because their partners died of AIDS. Especially among women, having been in successive marriages is also an important risk factor. Since successive marriages can result from either the death of a spouse or divorce, identification is problematic for this association. The association could be driven by reverse causality, as in the case of widowhood, or could be due to self-selection if individuals who are less likely to commit to one partner are also more likely to be infected by HIV. Nevertheless, this result, together with the finding that a substantial share of the population has been engaged in successive marriages, suggests that this group, especially women, could benefit from prevention programs targeted to their needs and vulnerabilities. Being in a polygynous union appears to be a risk factor for women.

Country-level regressions with HIV status as the dependent variable generally show results that are consistent with the pooled regression results (table 3 ). When a coefficient is significant in the pooled regression results, it generally has the same sign and significance in a substantial number of country-level regressions; for the remaining countries, it tends to be of the same sign but not significant, possibly because of sample size. For the variables for which the coefficient was not significant in the pooled regressions, there are only a few exceptions in which the coefficient is significant in the country-level regressions.

HIV prevalence is substantially higher in Cameroon (3.9 percent for men and 6.6 percent for women), Kenya (4.6 percent and 8.7 percent) and Tanzania (6.3 percent and 7.7 percent) than in Burkina Faso (1.9 percent and 1.8 percent) and Ghana (1.6 and 2.7 percent; bottom of table 3 ). Women are usually more likely to be HIV positive, except in Burkina Faso. Notice, however, that the age ranges in the survey are not the same for men and women except in Tanzania.

Contrary to the unadjusted means (table 5), but consistent with the pooled regression results (table 2), there is no significant association between HIV infection and years of education. Analyzing the same countries and data sets, Fortson (2008) finds a positive association between education and HIV infection. However, she does not control jointly for education and wealth and therefore her coefficients on the education variables are likely to reflect the confounded influence of education and wealth. Fortson also argues that the relationship between HIV infection and education is not linear, and so she uses a quadratic function of education.

To test the nonlinearity of the relationship, two specifications are included in which education is not constrained to be linear (table S2). Regression 4 includes education and education squared, as in Fortson (2008), and regression 5 , which does not to assume a functional form, include indicators for the 
education categories (no education, at least some primary education, and at least some secondary education or above). Overall, while there is some evidence of nonlinearity, it is difficult to conclude that there would be a strong association between education and HIV infection.

HIV infection is positively associated with urban status for women in Cameroon, men in Kenya, and both men and women in Tanzania. This result is consistent with that for the pooled regression (table 2). There is a strong positive association between being formerly married and HIV infection for women in Cameroon, Ghana, Kenya, and Tanzania. This association is also found in the pooled regressions and could be due to reverse causality.

Successive marriages seem to be an important risk factor. Consistent with the pooled regression results, having been in successive marriages is positively associated with HIV infection for women in Cameroon, Ghana, and Tanzania and for men in Tanzania. Polygyny does not seem to be associated with HIV infection, except in Burkina Faso, where the association is negative, and for women in Kenya, where the association is positive. The results for polygyny are different in the pooled regressions, which show a significant positive association for women. It might be that the significance is higher in the pooled regressions because of the larger sample size.

HIV test results are missing for some individuals who were randomly selected to be tested in the survey, because they refused to be tested or were absent or because of a technical problem. The proportion of people being tested (reported at the bottom of table S1) is always above 82 percent, but is higher (above 92 percent) in Burkina Faso and Cameroon and for women in Ghana. Refusal to be tested is the main reason for the absence of a test. The absence of a test result, if not random, might cause a bias. Table S1 analyzes the determinants of the likelihood to be tested if selected in the HIV sample of the surveys. Gersovitz (2007) also points out that some people, although randomly selected to be included in the sample, did not participate at all in the surveys (questionnaire and HIV test).

Mishra and others (2008) conclude that the potential bias due to nonresponse does not significantly affect the seroprevalence estimates obtained from nationally representative samples. However, for this analysis of the link between education and HIV infection, it is useful to verify whether the absence of an HIV test is correlated with education and other variables. Education does not seem to be associated with acceptance of the test (table S1). ${ }^{13}$ People in urban areas are generally less likely to be tested. ${ }^{14}$

13. In the marginal probit specification, there are two negative coefficients (men in Cameroon and in Kenya) that are significant at the 10 percent level.

14. Since HIV prevalence is generally higher in urban areas (tables 3 and 4), this could imply a slight downward bias in the estimates of overall HIV prevalence as well as a potential bias of the coefficients on urban location. This would require unobserved variables to be correlated, but not fully, with observed variables (HIV infection and urban location). However, overall, the facts that the coverage of the surveys is very good and that there is no association between education, the main variable of interest, and the probability of being tested in the survey, limits the scope for bias. 


\section{Sexual Behaviors and Attitudes Related \\ TO THE HIV/AIDS EPIDEMIC}

A range of sexual behaviors assumed to have an influence on the risk of HIV infection (condom use, extramarital sex, abstinence, virginity, and age at sexual initiation) are examined (tables 4 and S2-S5). Filmer (1998) and Blanc (2000) use earlier Demographic and Health Surveys to study the socioeconomic correlates of sexual behavior. Filmer generally finds a positive association between condom use outside marriage and education and urban status. Blanc finds that educated people are more likely to engage in nonregular sex but that they are also more likely to use condoms within those relationships. Two studies by de Walque $(2003,2007 \mathrm{a})$ analyze sexual behaviors for a cluster of villages in rural Uganda. Those sexual behaviors are at the heart of most prevention efforts, including the so-called "ABC" strategy (abstain, be faithful, or use a condom). The present study also analyzes the use of voluntary counseling and testing facilities, the probability that spouses will discuss AIDS, and the knowledge that an asymptomatic person can be HIV positive.

\section{Condom Use and Extramarital Sex}

The Demographic and Health Surveys ask respondents whether they used a condom during their last sexual intercourse and whether that intercourse occurred with a spouse or with another partner. Condom use is recommended in both cases, but not using a condom outside marriage is considered riskier. Because condom use at last intercourse differs widely according to whether the last intercourse was inside or outside marriage (compare the means in tables S3a and S3b), the cases are analyzed separately.

Table 4 uses pooled regressions as in table 2 to look at the association between education and condom use during marital and nonmarital sex and extramarital sex. Education is always positively associated with condom use, both with the spouse and with an extramarital partner. ${ }^{15}$ But among women, education is also positively associated with extramarital sex. ${ }^{16}$ Extramarital sex increases the risk of contracting HIV, but that risk can be mitigated with condom use. It might be because of these contradicting associations that education is not significantly associated with HIV status.

Examining the same three behaviors using country-level regressions finds that the positive association between education and condom use in marriage is robust and consistent with the pooled regression results (only for men in Burkina Faso and Kenya and for both genders in Tanzania is it not significant under the linear model; table S3). Education increases the likelihood of using

15. Extramarital sex includes all sexual relationships outside the union, regardless of whether an individual is married.

16. Under the probit specification, education is sometimes also positively associated with extramarital sex for men. 
a condom in extramarital relationships everywhere, except in Kenya (table S3b). Because condoms are an effective method of preventing HIV/AIDS, these findings support increasing access to education as a path to addressing the HIV/AIDS crisis in Africa.

The country-level regressions also indicate that education is positively associated with extramarital sex in several countries, for both men and women (table S3c). This association is consistent with the pooled regression results in table 4.

\section{Other Sexual Behaviors and Attitudes Related to HIV/AIDS}

Abstinence is another strategy for avoiding AIDS. The analysis shows that in several countries more educated people are less likely to abstain from sex (table S4a). Education is negatively associated with abstinence for both men and women in Burkina Faso and Cameroon, for men in Ghana, and for women in Tanzania (linear model). The analysis of virginity is done for singles only since it is assumed that all ever-married individuals have had sexual activity (the data confirm this). Education is positively associated with virginity among women in Ghana, Kenya, and Tanzania (table S4b). Only among men in Cameroon is there a negative association between virginity and education.

It is generally assumed that a later age at of sexual initiation is a way to prevent HIV/AIDS infection. ${ }^{17}$ Education is always associated with a later sexual debut for women (table S4c). This relationship might also be affected by reverse causality since pregnancy frequently means that a girl has to drop out of school. Education is also positively associated with age of sexual debut for men in Tanzania, but educated men in Burkina Faso and Cameroon have earlier sexual experiences.

Other attitudes and practices that are not sexual behaviors but are related to the HIV/AIDS epidemic were also examined. Education is always positively associated with obtaining information about one's HIV status (table S5a), with an increased level of discussion about AIDS between spouses (table $\mathrm{S} 5 \mathrm{~b})$, and with the knowledge that a healthy-looking person can be HIV positive (table S5c), used as an indicator of knowledge about the HIV/AIDS epidemic.

From this analysis, education appears to be one of the most consistent predictors of behavior and knowledge. Education predicts protective behaviors such as condom use, use of counseling and testing, discussion of AIDS between spouses, and knowledge of HIV/AIDS, but it also predicts a higher level of infidelity and a lower level of abstinence. It might be because of these contradicting associations that education is not significantly associated with HIV status.

17. Gersovitz (2005) shows several inconsistencies in self-reported age at first sexual intercourse by comparing subsequent Demographic and Health Surveys in the same countries. The results of this analysis should therefore be treated with caution. 


\section{Conclusions}

The last wave of Demographic and Health Surveys in many countries, especially in Africa, includes HIV testing for a representative sample of the population. A very useful addition, this testing allows for a better assessment of the epidemic in each country. However, as antiretroviral treatment is scaled up in many countries, HIV prevalence will become an ambiguous indicator. If prevalence is increasing, it will it become difficult to ascertain whether that is due to a higher HIV incidence, and therefore a failure of prevention efforts, or to lower AIDS-related mortality, and therefore to the success of treatment programs. The development of nationally representative measures of HIV incidence should therefore be encouraged. It would also be useful to include questions about antiretroviral treatment in the next wave of Demographic and Health Surveys.

This article takes advantage of the HIV data in the Demographic and Health Surveys to study the socioeconomic determinants of HIV status and sexual behavior in five African countries and, because the variables are defined similarly, to use pooled data to draw some interesting generalizations. While having the results of HIV testing, an objective biomarker, is a benefit of the new wave of Demographic and Health Surveys, a limitation is that sexual behaviors are self-reported. Another shortcoming of the analysis is that each of the five data sets is a cross section and many of the variables are potentially endogenous, although the most obviously endogenous variables, such as sexual behaviors, were not used as regressors. Thus, the coefficients in this study should not be taken to imply causal relationships. But even if causal links cannot be established, some reported associations clearly show that some categories of the population are at greater risk and specific prevention interventions should be directed to them.

Several findings can be generalized and are important for policymakers engaged in controlling the HIV/AIDS epidemic. Successive marriages are a significant risk factor, especially for women. Even if this result is due to selfselection, it suggests a need for specific prevention efforts for that group. Contrary to the evidence derived from unadjusted means, education is not positively associated with HIV status. But schooling is one of the most consistent predictors of behavior and knowledge: educational achievement predicts protective behaviors such as condom use, HIV testing, discussion of AIDS between spouses, and knowledge about HIV/AIDS. However, it also predicts a higher level of extramarital sex and a lower level of abstinence. It is possible that these associations cancel each other out, thus explaining why education is not significantly associated with HIV status.

However, while it is difficult to isolate a negative relationship between education and HIV in the overall population, a negative association can be measured in urban settings (table 2, regressions 7 and 8). It could be that the negative gradient between HIV infection and education takes time to develop 
and is found earlier in cities, where information spreads faster and where HIV prevalence is generally higher.

\section{ACKNOWLEDGMENTS}

The author thanks Quy-Toan Do, Timothy Johnston, Ted Miguel, Mead Over, and seminar and conference participants at the World Bank, the University of California at Berkeley, and the International AIDS Economics Network Conference in Cuernavaca, Mexico, for useful discussions, and Rachel Kline for editorial assistance.

\section{Supplementary Data}

A supplemental appendix to this article is available at http://wber. oxfordjournals.org/.

\section{REFERENCES}

Blanc, Ann K. 2000. The Relationship between Sexual Behavior and Level of Education in Developing Countries. Geneva, Switzerland: The Joint United Nations Programme on HIV/AIDS.

Burkina Faso Government. 2006. "Comité Ministériel de Lutte Contre le Sida." Ouagadougou. Unpublished report.

Burkina Faso Government and ORC Macro. 2004. Enquête Démographique et de Santé du Burkina Faso 2003. Ouagadougou: Institut National de la Statistique et de la Démographie.

Buvé, Ann, Michael Caraël, and Rea Hayes. 2001. "Multicentre Study on Factors Determining Differences in Rate of Spread of HIV in Sub-Saharan Africa: Methods and Prevalence of HIV infection." AIDS 15(suppl. 4):S5-S14.

Cameroon Government and ORC Macro. 2004. Enquête Démographique et de Santé du Cameroun 2004. Yaoundé: Institut National de la Statistique.

de Walque, Damien. 2003. "How Do Information and Education Affect Health Decisions? The Cases of HIV/AIDS and Smoking." PhD Dissertation, University of Chicago, Chicago, Illinois.

. 2006. Who Gets AIDS and How? The Determinants of HIV Infection and Sexual Behaviors in Burkina Faso, Cameroon, Ghana, Kenya and Tanzania. Policy Research Working Paper 3844. World Bank, Washington, DC.

2007a. "How Does the Impact of an HIV/AIDS Information Campaign Vary with Educational Attainment? Evidence from Rural Uganda." Journal of Development Economics 84(2):686-714.

—. 2007b. "Sero-Discordant Couples in Five African Countries: Implication for Prevention Strategies." Population and Development Review 33(3):501-23.

de Walque, Damien, Jessica S. Nakiyingi-Miiro, June Busingye, and Jimmy A. Whitworth. 2005. "Changing Association between Schooling Levels and HIV-1 Infection over 11 Years in a Rural Population Cohort in South-West Uganda." Tropical Medicine and International Health 10(10):993-1001.

Filmer, Deon. 1998. The Socio-Economic Correlates of Sexual Behavior: A Summary of Results from an Analysis of DHS Data." In Martha Ainsworth, Lieve Fransen, and Mead Over, eds. Confronting AIDS: Evidence from the Developing World. Brussels: European Commission.

Fortson, Jane G. 2008. "The Gradient in Sub-Saharan Africa: Socioeconomic Status and HIV/AIDS." Demography 45(2):303-22. 
Fylkesnes, Knut, Rosemary Mubanga Musonda, Kelvin Kasumba, Zacchaeus Ndhlovu, Fred Mluanda, Lovemore Kaetano, and Chiluba C. Chipaila. 1997. "The HIV Epidemic in Zambia: Socio-Demographic Prevalence Patterns and Indications of Trends among Childbearing Women.” AIDS, 11(3):339-45.

Fylkesnes, Knut, Rosemary M. Musonda, Moses Sichone, Zacchaeus Ndhlovu, Francis Tembo, and Mwaka Nonze. 2001. "Declining HIV Prevalence and Risk Behaviours in Zambia: Evidence from Surveillance and Population-Based Surveys.” AIDS 15(7):907-16.

Gersovitz, Mark. 2005. "The HIV Epidemic in Four African Countries Seen through the Demographic and Health Surveys." The Journal of African Economies 14(2):191-246.

2007. "HIV, ABC, and DHS: Age at First Sex in Uganda." Sexually Transmitted Infections $83(2): 165-68$.

Ghana Government and ORC Macro. 2004. Ghana Demographic and Health Survey 2003. Accra: Ghana Statistical Service and Noguchi Memorial Institute for Medical Research.

Hargreaves, James R., and Judith R. Glynn. 2002. "Educational Attainment and HIV-1 Infection in Developing Countries: A Systematic Review." Tropical Medicine and International Health $7(6): 489-98$.

Kenya Government and ORC Macro. 2004. Kenya Demographic and Health Survey 2003. Nairobi: Central Bureau of Statistics and Ministry of Health.

Kilian, Albert H.D., Simon Gregson, Bannet Ndyanabangi, Kenneth Walusaga, Walter Kipp, Gudrun Sahlmuller, Geoffrey P. Garnett et al. 1999. "Reductions in Risk Behaviour Provide the Most Consistent Explanation for Declining HIV-1 Prevalence in Uganda.” AIDS 13(3):391-98.

Mishra, Vinod, Bernard Barrere, R. Hong, and S. Kahn. 2008. "Evaluation of Bias in HIV Seroprevalence Estimates from National Household Surveys." Sexually Transmitted Infections 84(S1):i63-i70.

Morgan, Dilys, Cedric Mahe, Billy Mayanja, Martin J. Okongo, Rosemary Lubega, and James A.G. Whitworth. 2002. "HIV-1 Infection in Rural Africa: Is There a Difference in Median Time to AIDS and Survival Compared with That in Industrialized Countries?” AIDS 6(4):597-603.

Nicolas, Nagot, Amadou Ouangré, Abdoulaye Ouedraogo, Michel Cartoux, Pierre Huygens, Marie Christine Defer, Tarnagda Zékiba, Nicolas Meda, and Philippe Van de Perre. 2002. "Spectrum of Commercial Sex Activity in Burkina Faso: Classification Model and Risk of Exposure to HIV." Journal of Acquired Immune Deficiency Syndromes 29(5):517-21.

Nunn, Andrew J., Jane F. Kengeya-Kayondo, Sam S. Malabar, Janet A. Seeley, and Daan W. Mulder. 1994. "Risk Factors for HIV-1 Infection in Adults in a Rural Ugandan Community: A Population Study." AIDS 8(1):81-86.

Tanzania Government and ORC Macro. 2005. Tanzania HIV/AIDS Indicator Survey 2003-04. Dar es Salaam: Tanzania Commission for AIDS and National Bureau of Statistics.

UNAIDS (Joint United Nations Programme on HIV/AIDS). 2007. AIDS Epidemic Update: December 2007. Geneva: Joint United Nations Programme on HIV/AIDS.

WHO (World Health Organization) and UNAIDS (Joint United Nations Programme on HIV/AIDS). 2005. Progress on Global Access to HIV Antiretroviral Therapy. An Update on "3by5": June 2005. Geneva: World Health Organization. www.who.int/3by5/fullreportJune2005.pdf. 